\title{
Um sistema de atendimento remoto usando a língua brasileira de sinais
}

\author{
A remote helpdesk system using the Brazilian sign language
}

\section{Arthur Inácio do Nascimento ${ }^{1}$ orcid.org/0000-0001-5871-344X}

\section{João Henrique Correia Pimentel ${ }^{2}$ orcid.org/0000-0002-7441-0796}

${ }^{1}$ Escola Politécnica de Pernambuco, Universidade de Pernambuco, Recife, Brasil,

2 Universidade Federal Rural de Pernambuco, Pernambuco, Brasil.

E-mail do autor principal: Arthur Inácio do Nascimento arthur.inas@gmail.com

\section{Resumo}

No Brasil, aproximadamente 5,1\% da população apresenta algum tipo de deficiência auditiva. Grande parte dessa população utiliza a Língua Brasileira de Sinais (LIBRAS) para se comunicar, pois apenas uma pequena parcela apresenta alguma capacidade de expressão e recepção verbal. De acordo com a Lei Brasileira de Inclusão (LBI), a acessibilidade é direito de todos os indivíduos com algum tipo de deficiência, exigindo que esse grupo tenha acesso à informação em igualdade com as demais pessoas. Instituições públicas e de uso coletivo precisam proporcionar meios para que essa inclusão de fato aconteça. Este artigo propõe um sistema web de atendimento ao cliente surdo que se encontre em locais públicos como departamentos de trânsitos, agências bancárias, correios e aeroportos. O sistema atua como um intermediário na comunicação entre o indivíduo surdo e um intérprete de LIBRAS. Os resultados da avaliação apontam que o sistema atende às necessidades de comunicação entre surdos e ouvintes usando LIBRAS. Não obstante, foram identificados alguns pontos de aprimoramento e potenciais novas funcionalidade. Espera-se que este tipo de sistema possa impactar positivamente no cotidiano dos indivíduos surdos.

Palavras-Chave: LIBRAS; Sistema de Comunicação; Tecnologia Assistiva; Surdez;

\begin{abstract}
Approximately $5.1 \%$ of the population has hearing impairment in Brazil. The Brazilian Sign Language (LIBRAS) is used by deaf individuals to communicate, because only a small portion of this population has the capacity to receive and transmit information verbally. According to the Brazilian Inclusion Law (LBI), accessibility is a right of all individuals with disability. It ensures this group to have access to information on an equal basis with all people. And declares that public institutions must provide means for this inclusion to take place. This article proposes a web deaf customer service system to be used in places such as traffic departments, banks, post offices and airports. The system acts as a communication bridge between the deaf individual and a LIBRAS interpreter. The results of the evaluation indicate that the system has achieved its proposal of establishing communication between deaf and hearing individuals using LIBRAS. Enhancement and possible new features have already been noticed. Therefore, the system has a potential to positively impact the deaf customer service in everyday situations.
\end{abstract}

Key-words: Brazilian Sign Language, Communication System, Assistive Technology, Deaf People; 


\section{Introdução}

A comunicação é base fundamental na interação humana. O indivíduo ouvinte (IO) frequentemente se comunica através da fala utilizando termos oraisauditivos. Por outro lado, o indivíduo surdo (IS) em geral não consegue interagir dessa mesma maneira, devido à dificuldade na aquisição da linguagem oral decorrente da deficiência auditiva. O conjunto de elementos linguísticos usados pelos ISs são os manuais, corporais e faciais [1]. Essa incompatibilidade de modalidades dificulta a integração entre surdos e ouvintes, criando uma barreira de comunicação entre esses dois grupos.

Segundo o Censo de 2010 do Instituto Brasileiro de Geografia e Estatística (IBGE), existem aproximadamente 9,7 milhões de deficientes auditivos no Brasil [2]. Dentre eles cerca de 2 milhões possuem deficiência auditiva severa ${ }^{1}$, enquanto os demais 7,5 milhões apresentam alguma dificuldade auditiva de menor intensidade [3]. A LIBRAS ou Língua Brasileira de Sinais, é a língua materna dos surdos brasileiros e a segunda língua oficial do Brasil [4]. Para uma parcela dos surdos no Brasil, não existe 0 entendimento da língua portuguesa. Isto acontece porque há uma defasagem no aprendizado do português [5]. Desta maneira, escrever e ler em português se torna muito difícil para os ISs [6] [7], o que torna a LIBRAS sua única forma de comunicação possível.

Considerando-se as necessidades específicas da população com algum grau de deficiência, a Lei Brasileira de Inclusão (Lei 13.146/2015) vem afirmar a autonomia para que essas pessoas exerçam seus direitos de cidadania e de participação social em igualdade com as demais pessoas [8]. Essa lei estabelece que instituições públicas, empresas prestadoras de serviços públicos e empresas de uso coletivo são obrigadas a oferecer atendimento ao público com deficiência, incluindo-se o público com deficiência auditiva. Esses tipos de estabelecimentos devem disponibilizar recursos, sejam humanos ou tecnológicos, para garantir o atendimento dessa parcela da população.

Diante deste cenário, é possível afirmar que um intérprete de LIBRAS se torna imprescindível para intermediar diálogos entre IOs e ISs [9]. A dificuldade se torna evidente quando um IS precisa ser atendido presencialmente. Para isso, existe a necessidade por parte do IS em levar uma pessoa de confiança que possua noções básicas de LIBRAS ou mesmo contratar um intérprete para lhe ajudar a realizar uma atividade específica, como conseguir a carteira de habilitação ou negociar uma dívida no banco.

Com o intuito de amenizar o problema encontrado diariamente por pessoas surdas, e permitir que as instituições de grande porte atendam à legislação vigente, é proposto neste trabalho um sistema de atendimento remoto ao cliente surdo. O objetivo deste artigo é descrever um sistema que utiliza a LIBRAS para comunicação entre um usuário (IS), que esteja no estabelecimento, e um atendente remoto que possui conhecimentos em LIBRAS. Para descrever os requisitos do sistema e mostrar as interações das partes envolvidas, é utilizada a linguagem $i *$. A validação da proposta foi feita através de testes de aceitação com indivíduos surdos e com atendentes de LIBRAS.

Diferentemente de sistemas de videochamada convencionais, o sistema proposto se preocupa com características como: funcionamento em uma central de atendimento, de forma a diminuir a ociosidade dos atendentes; uso de um avatar 3D para representar diferentes atendentes de LIBRAS; registro do histórico de atendimentos; e baixo consumo de banda de Internet durante as chamadas.

Este artigo está dividido da seguinte maneira. Na seção 2 é apresentada a base deste trabalho, a saber: uma visão geral da Língua Brasileira de Sinais (LIBRAS); ferramentas de tradução portuguêsLIBRAS; e a linguagem de modelagem $i * 2.0$. Na seção 3 estão descritos os principais requisitos e a arquitetura do sistema proposto. Na seção 4, são apresentados os resultados preliminares referentes à validação do sistema proposto. $\mathrm{Na}$ seção 5 , são descritos os trabalhos relacionados, e na seção 6 , é apresentada a conclusão do trabalho.

\footnotetext{
${ }^{1} \mathrm{~A}$ surdez é classificada em 5 níveis, a depender da intensidade da perda auditiva: leve, moderada, acentuada, severa ou profunda. A perda total da audição é denominada anacusia [27].
} 


\section{Fundamentação teórica}

\section{$2.1 \quad$ LIBRAS}

A LIBRAS é a língua de sinais usada pelas comunidades de surdos brasileiras e a segunda língua oficial do Brasil [10]. Ela foi reconhecida no território brasileiro em abril de $2002 \mathrm{com}$ a Lei no 10.436. Esta língua apresenta uma gramática própria e utiliza mecanismos fonológicos, morfológicos, sintáticos e semânticos. O conjunto de elementos linguísticos usados na LIBRAS é formado por: configuração de mãos, ponto de articulação, movimentação, orientação e expressão corporal. Esses parâmetros são estruturados e usados simultaneamente no espaço durante os diálogos [1]. Enquanto o emissor está sinalizando em LIBRAS, o receptor utiliza apenas a visão para compreender o que está sendo comunicado.

É possível também representar as sinalizações em LIBRAS através de textos em português usando glosas de sinais. Essas glosas são formadas por uma ou mais palavras grafadas em maiúsculo semanticamente equivalentes à uma língua oral [11]. Este recurso é usado largamente em transcrições de conteúdos em português. A glosa passa a mesma ideia do texto original e pode ser exemplificada na seguinte frase em português: "Eu gosto de laranja", que se transforma em "EU GOSTAR LARANJA" quando grafada como glosa. A Figura 1 mostra os sinais em LIBRAS dessa glosa feitos por um avatar 3D.

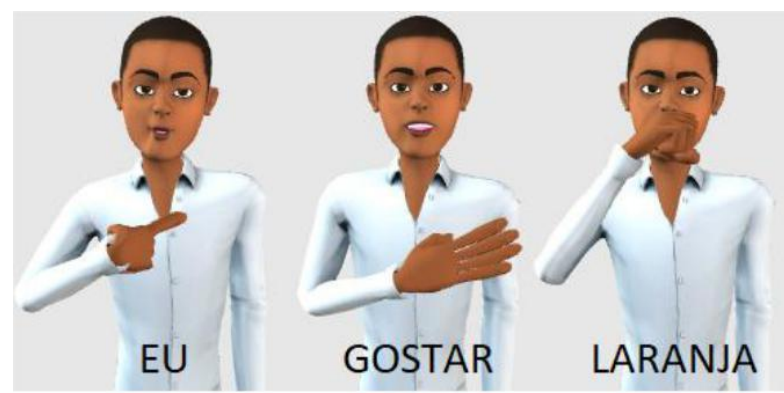

Figura 1: Avatar da ProDeaf sinalizando em LIBRAS. Fonte:www.weblibras.com.br

\subsection{Ferramentas de Tradução}

Com o intuito de tornar conteúdos digitais acessíveis para surdos, foram desenvolvidas ferramentas de tradução automática do português para a Língua Brasileira de Sinais. HandTalk [12], ProDeaf [13], Ribená [14] e VLibras [15] são exemplos de Tecnologias Assistivas (TAs) que possuem ferramentas para tradução de textos em português disponíveis em páginas web [16]. O texto enviado para essas ferramentas podem ser uma frase em português ou até mesmo uma glosa. 0 conteúdo passa por um engenho de tradução, onde é processado e convertido em uma sequência de sinais em LIBRAS. A sinalização propriamente dita é feita por um avatar 3D carregado dentro de um navegador web. Essas ferramentas também oferecem uma Application Programming Interface (API) para que o responsável pelo website consiga prover uma maior interação com 0 personagem virtual.

Por si só as ferramentas citadas não resolveriam o problema tratado neste artigo, visto que não existe uma maneira dessas ferramentas processarem diretamente o que foi enviado pelo IS e retornarem uma resposta em LIBRAS de forma autônoma. O funcionamento das ferramantas de tradução acontece de forma reativa, ou seja, a sinalização em LIBRAS acontece apenas se houver um input através do envio de um texto em português.

\subsection{O Framework $i * 2.0$}

Como o sistema aqui proposto é um sistema sócio-técnico, optou-se por adotar o framework $i^{*}$ para representar os requisitos do sistema. O $i^{*}$ (pronuncia-se i-star, ou i-estrela), originalmente 
proposto por Eric Yu [17], é usado para modelar sistemas com base em atores e objetivos (goals). 0 principal intuito do framework é representar a interação entre atores, objetivos que devem ser alcançados, especificar tarefas, recursos, relacionamentos e dependências. $O i^{*}$ foi adotado pela comunidade acadêmica principalmente nas áreas de engenharia de requisitos e modelagem de negócios [18]. A versão mais recente do framework é o $i * 2.0$, que traz mudanças em alguns elementos da linguagem na tentativa de mitigar limitações da versão inicial [19].

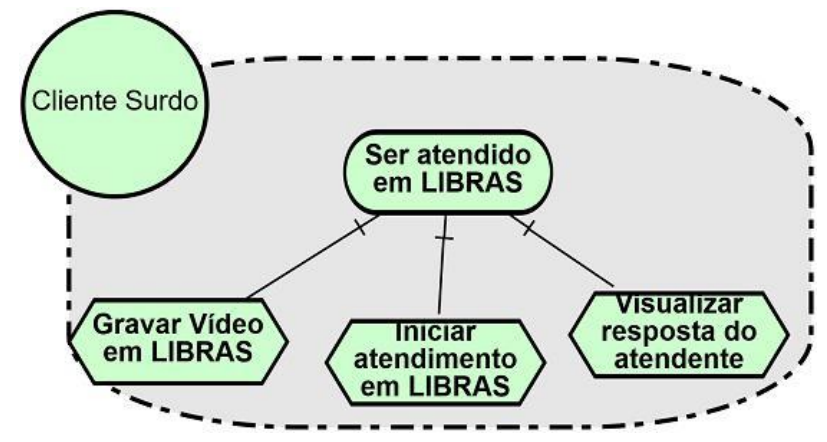

Figura 2. Exemplo de modelo da linguagem $i * 2.0$

O principal elemento do modelo é o Ator, que é representado graficamente como um círculo e suas limitações são definidas em uma área cinza delimitada por uma linha tracejada. Dentro desta área, são descritos as inter relações e elementos que o ator quer ter ou desempenhar. Os elementos suportados na linguagem são: objetivo (goal), atividade (task), qualidade (quality) e recurso (resources). A Figura 2 descreve $o$ ator Cliente Surdo que tem como objetivo Ser atendido em LIBRAS. Para que isso ocorra, é preciso desempenhar as seguintes atividades: Iniciar atendimento em LIBRAS, Gravar vídeo em LIBRAS e Visualizar resposta do atendente.

\section{Sistema de atendimento remoto usando a LIBRAS}

O sistema proposto visa proporcionar maior autonomia aos indivíduos surdos (IS), permitindo que eles interajam com representantes de uma instituição através, unicamente, da Língua Brasileira de Sinais (LIBRAS).

A tecnologia assistiva aqui apresentada é uma aplicação web de atendimento ao cliente surdo. 0 sistema oferece atendimento virtual através da interação de um IS presente em um estabelecimento com um atendente proficiente em LIBRAS (AL). O IS interage com um terminal de autoatendimento no estabelecimento, em LIBRAS, enquanto os ALs estão localizados remotamente em uma central de atendimentos. O papel do AL é extremamente importante no processo de atendimento ao IS, pois ele possui o conhecimento em LIBRAS necessário para interagir de forma plena com o IS.

O fluxo de atendimento começa com o IS interagindo com o sistema através de um terminal de autoatendimento, o qual deve incluir uma câmera para que o usuário grave e envie vídeos da sua comunicação em LIBRAS. O AL recebe o vídeo e, utilizando seu conhecimento de LIBRAS, responde o IS usando glosas de sinais. A resposta é enviada de volta ao IS, que terá a visualização do conteúdo através da sinalização de um avatar 3D. Logo após encerrar o processo descrito, tanto o AL como o IS avaliam a experiência pontuando vários aspectos do atendimento.

Como os AL possuem experiência em LIBRAS, optouse por enviar glosas de sinais ao invés de textos em português. Desta maneira, o AL tem um controle maior sobre quais sinais o avatar vai sinalizar, deixando que a ferramenta de tradução reproduza exatamente o que foi enviado e diminuindo-se, assim, potenciais erros de comunicação.

A Figura 3 representa os requisitos do sistema, através da linguagem $i^{*}$. Os indivíduos que interagem com o sistema são o Cliente Surdo e o AtendenteIntérprete. Os outros atores que se relacionam com 0 Sistema de atendimento em LIBRAS são o ProDeaf Framework de Tradução e a própria Instituição. O principal objetivo do Cliente Surdo é Ser atendido em LIBRAS. Para isso é preciso Iniciar Atendimento em LIBRAS através do Totem de autoatendimento e uma chamada interativa é iniciada pelo Sistema de atendimento LIBRAS. O cliente utiliza a câmera do dispositivo para Gravar Vídeo em LIBRAS, que será enviado e processado pelo sistema. O sistema vai Enviar Dados ao Atendente, e em seguida, Reproduzir Vídeo enviado pelo cliente.

O Atendente Intérprete tem como objetivo principal Fornecer assistência em LIBRA ao Cliente Surdo. Para que isso ocorra 0 atendente foi devidamente cadastrado no sistema e possui credenciais de acesso válidas. A tela de chamada oferece uma visão geral ao atendente de qual 


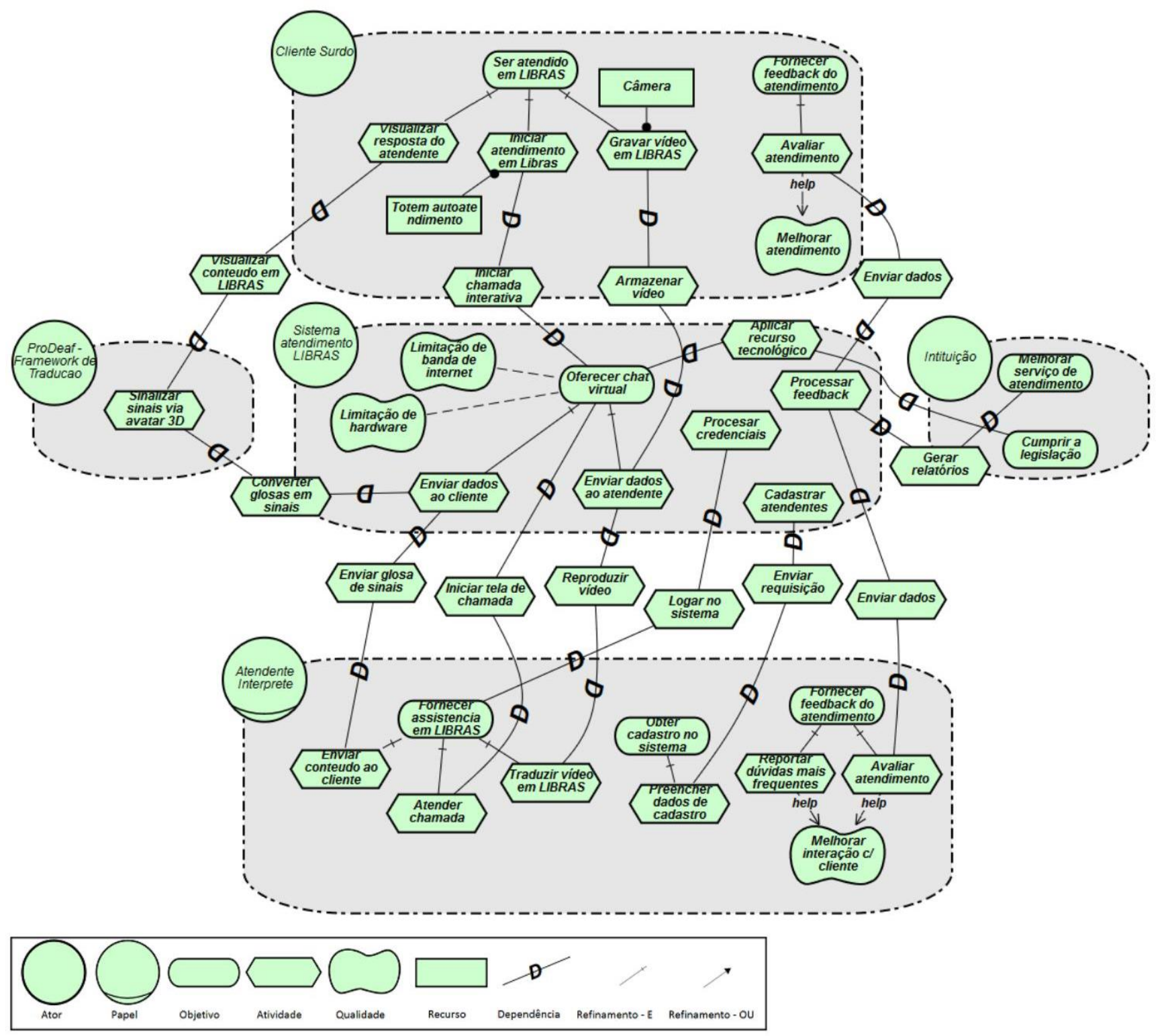

Figura 3. Sistema de atendimento em LIBRAS descrito na linguagem $i * 2.0$.

estabelecimento iniciou um atendimento. Após Atender a Chamada feita pelo Cliente Surdo, o atendente precisa Traduzir o Vídeo em LIBRAS, identificar a necessidade do IS e Enviar Conteúdo ao Cliente. As glosas são montadas e enviadas para o Sistema de atendimento LIBRAS, que por sua vez, se comunica com o ProDeaf - Framework de Tradução. A engine do framework será capaz de Converter glosas em sinais e Sinalizar sinais via avatar 3D. O Cliente Surdo pode Visualizar a Resposta do Atendente através do avatar sinalizando em LIBRAS. 5
A instituição por sua vez, precisa Cumprir a legislação aplicando o Sistema de atendimento em LIBRAS. Além disso, a instituição tem como objetivo Melhorar o serviço de atendimento oferecido aos clientes surdos. Para isso é preciso Processar Feedback tanto do Cliente Surdo quanto do Atendente Intérprete. Estes dois atores têm como objetivo secundário Fornecer Feedback do Atendimento para Gerar Relatórios relevantes para o aperfeiçoamento do sistema. 


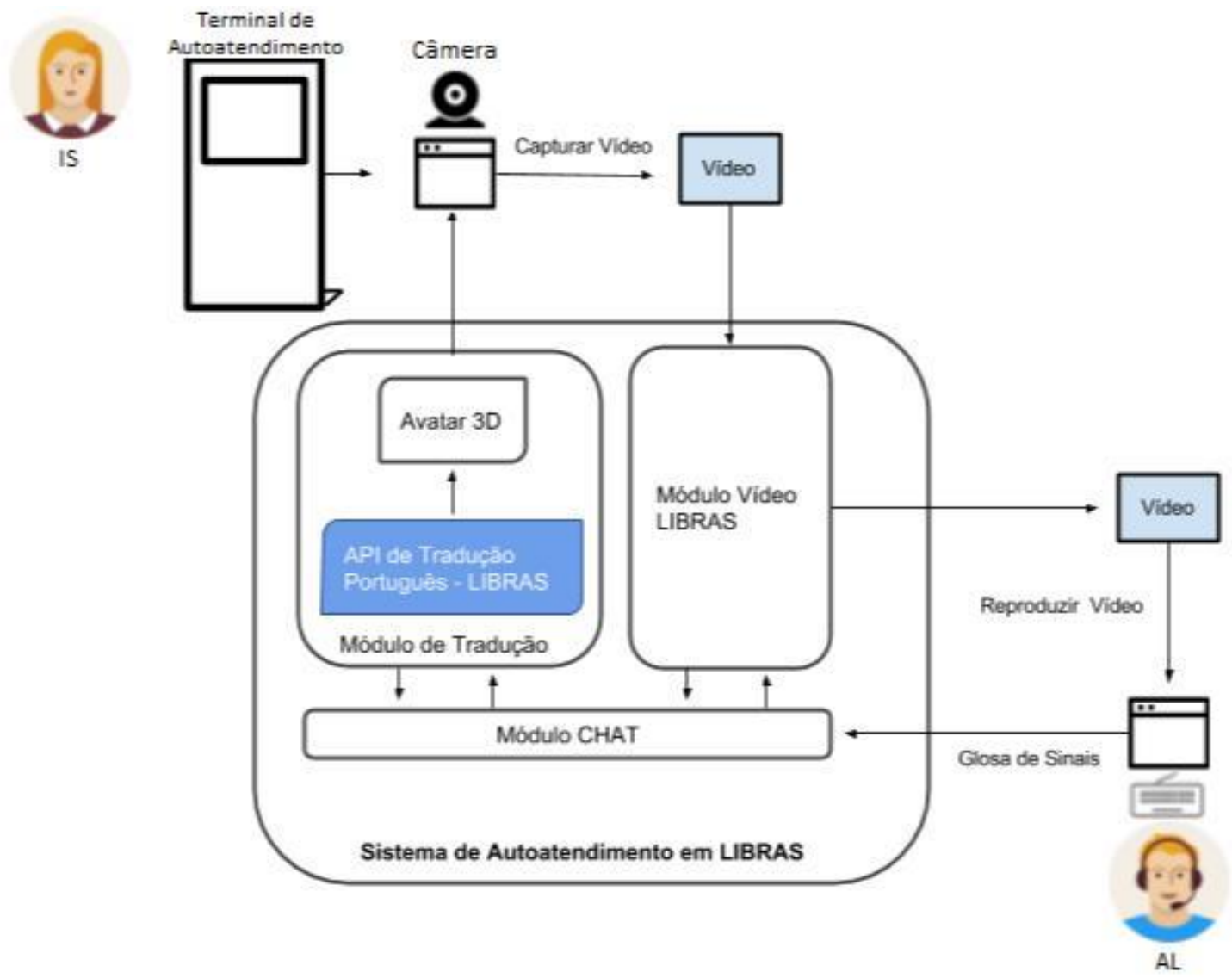

Figura 4. Visão geral do sistema

\subsection{Arquitetura do Sistema}

O sistema proposto é uma aplicação web que possui duas telas principais de comunicação: uma tela destinada ao IS e outra ao AL. Os componentes internos da aplicação são divididos em três módulos: Módulo Chat, Módulo de Vídeo LIBRAS e Módulo de Tradução, como representado pela Figura 4. Cada um desses módulos é apresentado nas subseções a seguir.

\subsubsection{Módulo Chat}

A comunicação entre o IS e o AL acontece em tempo real dentro da aplicação. Cada mensagem de texto ou vídeo trocado entre os usuários ocorre dentro de um canal de comunicação através da web. O objetivo é promover uma interação rápida e de baixa latência. Além disso é preciso que o IS se sinta confortável com a ferramenta e tenha uma experiência satisfatória durante todo o atendimento.
Websocket API é uma tecnologia usada para comunicação entre um navegador web e um servidor. Essa API consegue estabelecer uma conexão bidirecional em canal full-duplex sobre um único socket [20]. O padrão Websocket é um protocolo baseado em TCP (Transmission Control Protocol) com sua especificação definida no RFC 6455 [21]. Essa tecnologia foi usada no sistema proposto neste artigo.

Todas as mensagens trocadas usando o processo descrito são visualizadas pelo IS e pelo $A L$ em suas respectivas telas. Este módulo atua dentro do sistema de forma transversal dando suporte aos outros dois módulos.

A tela principal do sistema no lado cliente possui, à partida, apenas um botão para início do atendimento. Na primeira interação com o sistema, o IS recebe uma instrução em LIBRAS descrevendo os passos para iniciar a chamada. Após pressionar esse botão inicial, o IS espera o próximo AL disponível atender a chamada para começar a interação. Em sua tela, o IS possui uma área para gravação de vídeos (A), um avatar 3D para sinalização dos sinais (B), uma área de histórico de mensagens (C) e um campo de 
texto livre (D). A área de gravação de vídeos reflete a imagem gerada pela câmera vinculada ao terminal de autoadendimento. O IS visualiza sua própria imagem, e inicia a gravação e o término do vídeo através do toque na tela. O avatar 3D será responsável por sinalizar em LIBRAS o conteúdo enviado ao IS. A área de histórico de mensagens armazena os conteúdos das mensagens trocadas entre o IS e o AL. O campo de texto presente serve como opção secundária para envio de números como: RG, CPF, número de protocolo, número da conta, entre outros. Esses componentes são mostrados na Figura 5.

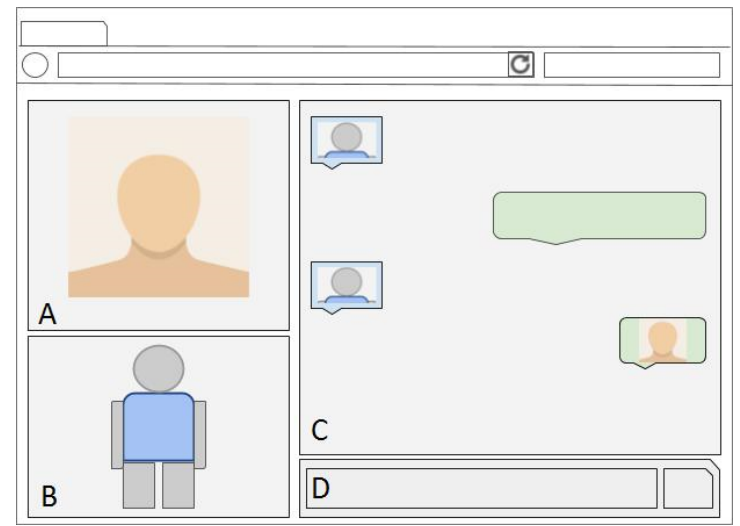

Figura 5. Tela do usuário (Indivíduo Surdo)

A tela do AL apresenta uma área com todas as chamadas que foram feitas usando o sistema proposto e que ainda não foram atendidas. Com esta abordagem, uma pequena quantidade de ALs pode atender usuários em diferentes localidades. O atendente ainda tem a possibilidade de atender ISs simultaneamente, usando diferentes telas de comunicação.

Baseado no conceito de fila (First In, First Out, em português, primeiro a entrar, primeiro a sair), O AL escolhe a próxima chamada para proceder com o atendimento. Uma nova tela será carregada para que a interação com o IS comece. Uma vez iniciado o atendimento, em sua tela, o AL possui um campo de texto no qual ele pode escrever a glosa que será enviada para o módulo de tradução (A), uma área de histórico de mensagens (B) e uma área para frases mais usadas durante o atendimento (C). O campo de texto possui duas opções de envio através de diferentes botões. O primeiro, e principal opção, envia a glosa de sinais ao IS. O segundo envia um texto puro que não será sinalizado automaticamente pelo avatar.
Esta opção é usada em casos que o AL precisa enviar algum número para IS, que será utilizado em um segundo momento. A área de histórico de mensagens se comporta da mesma maneira que na tela do IS. A área de frases mais usadas oferece de forma rápida frases prontas, que serão enviadas ao IS sem a necessidade da escrita das glosas. Esses componentes são mostrados na Figura 6.

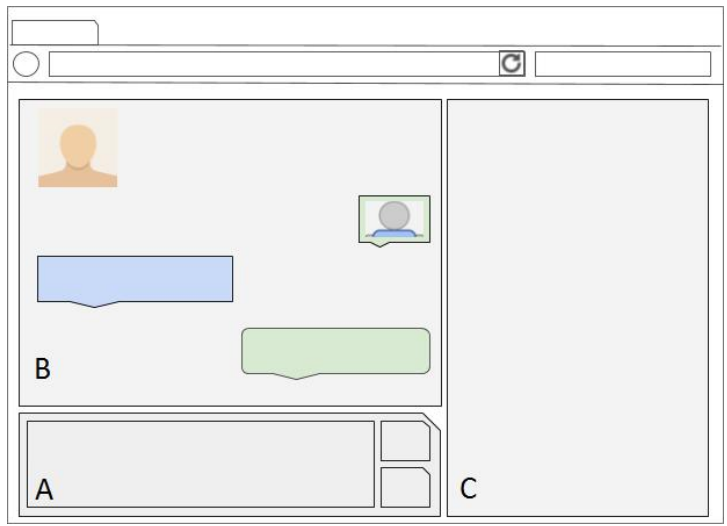

Figura 6. Tela do atendente (Intérprete)

\subsubsection{Módulo Vídeo LIBRAS}

Este módulo é responsável por utilizar a câmera conectada ao dispositivo para gravar toda a sinalização em LIBRAS do IS. Para que esse processo seja possível, é necessário um navegador web capaz de acessar e manipular recursos da câmera de vídeo. A API JavaScript MediaRecorder é nativa nos principais navegadores modernos, como Mozilla Firefox e Chrome, e pode ser usada para essa tarefa. Ela consegue capturar os dados referente a stream da câmera e gerar um arquivo de vídeo [12].

Depois de gravar o vídeo, é feita uma chamada REST (Representational State Transfer) do tipo POST ao servidor da aplicação enviando os dados resultante da gravação. Esse arquivo é acessado na página do $\mathrm{AL}$ dentro da área de histórico de mensagens. Para cada interação do IS com este módulo, um arquivo diferente é enviado ao servidor seguindo o processo descrito acima. Após analisar o vídeo recebido, o AL identifica a dúvida do IS e segue o fluxo de atendimento. 


\subsubsection{Módulo de Tradução}

Este módulo converte as glosas enviadas pelo $A L$ em sinais, que são visualizados através de um avatar 3D. O uso de um avatar, em detrimento ao envio contínuo de vídeos pelo $\mathrm{AL}$, foi selecionado por requerer menor consumo de banda de internet por parte do sistema. Outros pontos que influenciam essa decisão são: maior controle sobre a renderização do sinal, maior visibilidade dos movimentos executados pelo avatar e possibilidade de personalizar a aparência visual do avatar 3D.

A ferramenta de tradução de português para LIBRAS está integrada à interface gráfica do terminal de autoatendimento. No momento em que o texto contendo a glosa de sinais chega ao cliente, o sistema o envia diretamente ao framework de tradução para que seja feita a conversão para LIBRAS.

No sistema proposto foi usado o framework de tradução da ProDeaf. É preciso adicionar um script na página web do chat virtual para que ocorra o carregamento da ferramenta e a interação com o avatar 3D. O sistema de atendimento interage com o framework enviando textos (glosas) provenientes do AL. O framework da ProDeaf chama o seu próprio serviço de tradução que devolve um arquivo do tipo JSON [22] representando os sinais em LIBRAS. Por sua vez, o avatar 3D executa toda a sinalização baseada na resposta do serviço da ProDeaf, que é visualizada pelo IS em sua tela.

\section{Validação}

Este sistema de comunicação está sendo implementado por uma empresa brasileira de tecnologia assistiva, e está atualmente sendo homologado junto a uma grande empresa nacional. A avaliação realizada simula o atendimento de um IS com um AL baseado no contexto e processos do cliente. Abaixo estão os resultados dos testes preliminares do sistema em versão alpha realizados em parceria com a empresa em questão.

\subsection{Perfis dos testadores}

Os perfis dos testadores principais do sistema em sua versão alpha estão descritos a seguir. De modo a considerar as especificidades da Língua Brasileira de Sinais, os três sujeitos de teste possuem fluência no idioma Libras.
Sujeito A: Intérprete de Libras com 20 anos de atuação no ramo. Possui conhecimento intermediário de informática, com domínio pleno de técnicas de tradução de conteúdos em português por meio de glosa.

Sujeito B: Intérprete de Libras com aproximadamente 8 anos de atuação no ramo. Conhecimento avançado de informática, sem domínio de técnicas de tradução de conteúdos em português por meio de glosa.

Sujeito C: Indivíduo com surdez total desde o nascimento, professor universitário de Libras, com 30 anos de idade. Com conhecimento básico de informática.

\subsection{Roteiro de testes}

Os testes foram estruturados da seguinte forma:

(1) explanação dos objetivos do teste e funcionamento da ferramenta; (2) demonstração da ferramenta, de modo a guiar os testadores na interface; (3) pilotagem guiada do sujeito $A$, simulando o papel do Atendente Libras ( $A L$ ), e interação guiada por parte do sujeito $B$, simulando o papel do Indivíduo Surdo (IS); (4) pilotagem não guiada do sujeito $A$ no papel de AL com o sujeito $B$ no papel de IS; (5) inversão dos papéis entre os sujeitos A e B de forma não guiada; (6) Teste de aceitação com o sujeito $A$ atuando como (AL) e o sujeito $C$ atuando como usuário final do sistema no ambiente simulando uma operação de atendimento a um cliente surdo.

Os passos (1) e (2) do roteiro têm como objetivo apresentar de forma geral os fluxos do sistema e as funções dos componentes da inteface gráfica. $O$ passo

(3) apresenta ao Sujeito A a ferramenta na visão do atendente $(A L)$, que será responsável por atender 0 IS. Ainda nessa configuração, é apresentado ao Sujeito B a visão do sistema pelo IS. No passo (4) ocorre a comunicação entre o Sujeito A e o Sujeito B na mesma configuração anterior, com a diferença de não existir interferência externa. Os dois sujeitos constroem um diálogo de forma espontânea e exploratória. No passo (5) ocorre a inversão dos papéis entre o Sujeito $A$ e $B$, para respectivamente IS e AL. O objetivo da interação e inversão dos papéis é garantir que os usuários intérpretes tenham uma visão completa dos fluxos que o sistema oferece, facilitando a validação ao final dos testes. O passo (6) coloca o Sujeito A como o atendente e o Sujeito C como o IS. Este último passo mostra uma 
configuração que simula um atendimento real. $O$ objetivo é validar os requisitos do sistema e verificar se as necessidades de atendimento ao IS foram alcançadas.

Durante os processos acima, foram levantadas questões relacionadas ao uso do sistema. A seguir estão as principais perguntas que guiaram os resultados descritos na seção seguinte.

a) Há algum tipo de quebra de comunicação no decorrer da interação com o sistema?

b) Qual sua avaliação sobre o avatar 3D durante as sinalizações em LIBRAS?

c) O que você sentiu falta durante o processo de comunicação?

d) O sistema atendeu a expectativa quanto a comunicação entre um IS e um AL?

\subsection{Resultados do teste}

De acordo com os apontamentos realizados pelos sujeitos $A$ e $B$, foram identificados alguns pontos relevantes de melhoria. Esses pontos estão relacionados tanto a requisitos funcionais quanto a não funcionais do sistema, sendo eles:

- Pensando em uma comunicação assíncrona, ou seja, aquela onde $\mathrm{O} A \mathrm{~L}$ não precisa necessariamente enviar uma resposta ao IS de imediato, não há necessidade de apresentar algo para indicar visualmente uma possível espera. Do contrário, caso a comunicação aconteça de forma síncrona, ou seja, em que há fluidez comunicacional e o IS aguarda movimentação do avatar por parte da resposta do AL, é necessário que haja um botão de ação rápida que sinalize ao IS a necessidade de

"aguardar". Esse fato ficou evidenciado especialmente quando o sujeito (B) de teste, que não tinha fluência na ferramenta, interagiu com o sistema, especialmente em respostas mais longas que, portanto, demandam mais tempo para digitação da resposta;

- A quantidade de fluxos previstos no sistema é algo a ser pensado em algum modelo de síntese, como mapas mentais. Essa necessidade surge da importância que os intérpretes deram ao fato de conhecerem previamente palavras e frases relacionadas ao contexto. Esse fato ajudaria os intérpretes no momento da criação das glosas de sinais que serão enviadas ao IS. O fato é atenuado pela presença do recurso de auto complete de texto e pela área de frases rápidas da tela do $\mathrm{AL}$. Caso o intérprete digite uma palavra-chave no campo de digitação livre, o sistema é capaz de oferecer uma lista de palavras que começam com as letras digitadas.

- Foram apontados um total de 17 sinais, ou seja, palavras da LIBRAS, faltantes na plataforma, que eram necessários para uma comunicação mais efetiva e fluída. Apesar disso, o avatar foi capaz de sinalizar por meio da datilologia - digitação no ar com as mãos de cada letra da palavra que não constava cadastrada. Tal fato demonstra a capacidade do sistema de transmitir qualquer mensagem, porém a necessidade inicial de manutenção proativa e reativa;

- O IS, mesmo tendo apontado que entendeu toda comunicação sinalizada pelo avatar, sugeriu acentuar as expressões faciais (positivo / negativo / grande / pequeno) do avatar 3D.

- Devido a limitações de banda, o cliente surdo deve enviar pequenos vídeos de até $10 \mathrm{~s}$ com sua dúvida ou problema - isso foi encarado como um ponto de melhoria. O objetivo é tornar a comunicação um pouco mais fluída aumentando a duração dos vídeos.

- Percebeu-se a necessidade de um préatendimento ou triagem dos dados do cliente que está iniciando um novo atendimento.

- A fim de trazer elementos para melhoria contínua do processo, uma breve avaliação do atendimento e da qualidade dos sinais em Libras será apresentada ao indivíduo surdo que encerrou um atendimento.

- Se faz necessário uma auto calibragem da qualidade dos vídeos enviados do Cliente para o Atendente a fim de não esgotar a banda que já está, em alguns casos, saturada. 
- Por outro lado, os arquivos do tipo JSON de comando para o avatar na direção Atendente para o Cliente causaram impacto insignificante na saturação da banda de internet.

Além dos pontos acima levantados durante a avaliação da ferramenta, ocorreu também um retorno subjetivo: a expressão de encantamento por parte do sujeito $C$ em sua primeira interação com o avatar foi plena e emocionante. Os primeiros comentários foram "incrível, entendi tudo".

\section{Trabalhos relacionados}

Vários estudos foram realizados com o objetivo de ajudar a comunicação entre deficientes auditivos e indivíduos ouvintes nos mais diferentes contextos. Amarasinghe e Wimalaratne [23] mostram uma tecnologia assistiva para comunicação através do telefone celular. Com o auxílio do aparelho móvel, o IO fala e o conteúdo é convertido em texto e enviado para o celular do IS. No celular, ocorre a conversão do texto recebido para um feedback tátil usando uma versão abreviada do código Morse. A tecnologia se mostrou eficiente para a comunicação com deficientes auditivos usando frases curtas. Para usar este sistema os ISs devem ter o mínimo de conhecimento de código Morse e também precisam aprender a versão abreviada proposta neste artigo. O artigo também destaca a dificuldade de criar uma versão do código Morse abreviada.

Realidade aumentada (RA) e reconhecimento automático de voz também foram usados para facilitar a comunicação entre deficientes auditivos e ouvintes [24]. O narrador fala utilizando o microfone do computador, e um dos módulos do framework transforma aquela fala em texto. O conteúdo que foi convertido é apresentado ao surdo em forma de balões de diálogo em uma chamada de vídeo. Por sua vez, o indivíduo surdo digita a resposta e o framework transforma o texto em áudio e envia para o narrador. Desta forma, o narrador não precisa saber língua de sinais, mas o deficiente auditivo deve ter um conhecimento de escrita e leitura de textos.

Também foi encontrado na literatura uma tecnologia para comunicação entre IS e IO proposta para correios chamada TExt and Sign Support Assistant (TESSA) [25]. O objetivo daquele sistema é ajudar a comunicação entre um cliente surdo e o funcionário dos correios, convertendo as respostas faladas do funcionário em Língua Britânica de Sinais
(LBS). No entanto, foi reportado que mesmo com o sistema algumas tarefas podiam ser realizadas pelo IS independentemente do sistema. Alguns pontos de melhoria também foram levantados como: expressões faciais e aparência do avatar 3D. Além da latência entre a fala do funcionário até a sinalização feita pelo avatar 3D [25].

Como identificado nesses trabalhos, existe a necessidade do IS possuir um certo conhecimento da língua escrita (inglês), ou ainda de outras simbologias como o código Morse. O sistema proposto neste artigo se baseia apenas no conhecimento da LIBRAS do IS, sendo portanto potencialmente mais acessível.

Outro sistema foi criado com intuito de auxiliar os ISs que precisam renovar sua carteira de habilitação [26]. O sistema possui 2 módulos principais. $O$ primeiro converte a voz do usuário em LSE (Língua de Sinais Espanhola) e depois é feita a sinalização por um avatar 3D. O segundo módulo oferece uma interface para o indivíduo surdo montar uma glosa de sinais que será convertida em um áudio direcionado ao narrador. Ao final do trabalho, foi reportado pelos usuários a falta de naturalidade do avatar, além de pontos de melhoria na reprodução dos sinais em LSE. O ponto de melhoria reportado sobre o avatar 3D e seu desempenho durante as sinalizações também foi identificado na avaliação do nosso sistema.

\section{Conclusões}

Este artigo apresenta um sistema para auxiliar o cliente surdo que precisa usar serviços de atendimento ao consumidor em estabelecimentos públicos e de uso coletivo. A ideia principal é que o indivíduo surdo (IS) use seus conhecimentos de LIBRAS para interagir com um intérprete através de um chat virtual, e tenha suas demandas e dúvidas sanadas também por meio da LIBRAS. Ou seja, ele será capaz de se comunicar via um terminal de autoatendimento mesmo sem conhecimento da língua portuguesa. Como requisito não funcional do projeto, há o fato da limitação de banda e da economia de recursos de hardware. Sendo assim, partiu-se para uma abordagem baseada em avatar, na qual o atendente envia mensagens de texto (glosas) que são então traduzidas para sinais em LIBRAS e apresentados por meio de um avatar 3D, utilizando-se o framework de tradução da empresa ProDeaf.

Foram realizados testes controlados da ferramenta com dois sujeitos ouvintes especialistas em Libras, de 
modo a validar o quão os módulos atendem o objetivo final de comunicação. Com base nos testes, é possível concluir que a ferramenta cumpre seus objetivos comunicacionais. Dado que a comunicação é um processo orgânico, portanto vivo, percebe-se com os testes a necessidade de incrementos na ferramenta, principalmente quanto ao Módulo de Tradução. Tal necessidade se dá pelo fato de que o vocabulário precisa ser constantemente ampliado.

De acordo com os testes desenvolvidos, espera-se também que, após a entrada em produção e a interação com uma massa cada vez maior de usuários, o sistema necessite cada vez menos de incrementos, atingindo um ponto de maturidade tal que possibilite maior fluidez e autonomia dos operadores, representados no presente artigo na figura dos Atendentes Libras $(A L)$.

\section{Referências}

[1] M. Goebel e A. Z. Cordenonsi, "Ferramenta para a tradução da sintaxe da língua portuguesa para a língua brasileira de sinais", XII Simpósio Brasileiro de Informática na Educação, 2001.

[2] Instituto Brasileiro de Geografia e Estatística (IBGE), "Resultados preliminares da amostra", 2011.

Disponível em:

http://www.ibge.gov.br/home/estatistica. [Acesso em 10 Dezembro 2017].

[3] Portal Brasil, "Apesar de avanços, surdos ainda enfrentam barreiras de accessibilidade". Disponível em: http://www.brasil.gov.br/cidadania-. [Acesso em 10 Dezembro 2017].

[4] Diário Oficial da União, Brasil, Dispõe sobre a Língua Brasileira de Sinais - Libras e das outras providências, Brasília, DF: Seção 1, no 79, Lei no 10.436 de 24 de abril de 2002, p. 23.

[5] H. M. M. L. Salles, "Ensino de língua portuguesa para surdos: caminhos para a prática pedagógica", Brasil. Ministério da Educação. MEC/SEESP, 2002.

[6] M. C. d. C. Pereira, "O ensino do português como segunda língua para surdos: princípios teóricos e metodológicos", Educar em revista, no Especial 2, pp. 143157, 2014.
[7] C. B. F. d. Lacerda, "A inclusão escolar de alunos surdos: o que dizem alunos, professores e intérpretes sobre essa experiência", Cad. Cedes, vol. 26, pp. 163184, 2006.

[8] "Lei brasileira de inclusão da pessoa com deficiência". Disponível em: http://www.planalto.gov.br/ccivil_03/_at. [Acesso em 17 Dezembro 2017].

[9] Diário Oficial da União, Brasil, Regulamenta a profissão de tradutor e intérprete da língua brasileira de sinais - LIBRAS, Brasília, DF: Seção 1, no 169, Lei no 12.319, de $1^{\circ}$ de setembro de 2010.

[10] R. M. Quadros, Educação de surdos: a aquisição da linguagem, Artmed, 2009.

[11] L. McCleary e E. Viotti, "Transcrição de dados de uma língua sinalizada: um estudo piloto da transcrição de narrativas na língua de sinais brasileira (LSB),"

Bilingüismo dos surdos: Questões lingüísticas e educacionais, no Cânone Editorial, pp. 73-96, 2007.

[12] "Hand Talk. Uma solução digital para inclusão social". Disponível em: http://www.handtalk.me. [Acesso em 17 Dezembro 2017].

[13] "ProDeaf WebLibras - Tradutor de Sites". Disponível em: http://www.weblibras.com.br. [Acesso em 17 Dezembro 2017].

[14] "Rybena Inclusão Digital em LIBRAS e voz". Disponível em: http://portal.rybena.com.br/site-rybena. [Acesso em 17 Dezembro 2017].

[15] "VLibras Tradução de Português pra Libras". Disponível em: http://www.vlibras.gov.br/. [Acesso em 17 Dezembro 2017].

[16] R. Bersch, "Introdução à tecnologia assistiva", CEDI, 2008.

[17] J. Horkoff e E. Yu, "Comparison and evaluation of goal-oriented satisfaction analysis techniques" Requirements Engineering, vol. 18, pp. 199-222, 2013.

[18] J. Horkoff, T. Li, F.-L. Li, M. Salnitri, E. Cardoso, P. Giorgini, J. Mylopoulos e J. Pimentel, "Taking goal models downstream: a systematic roadmap", Research Challenges in Information Science (RCIS), 2014 IEEE Eighth International Conference on, pp. 1-12, 2014. 
[19] F. Dalpiaz, X. Franch e J. Horkoff, "iStar 2.0 language guide", arXiv preprint arXiv:1605.07767, 2016.

[20] The WebSocket Protocol - IETF Tools, "RFC 6455". Disponível em: https://tools.ietf.org/html/rfc6455. [Acesso em 17 maio 2017].

[21] MediaStream Recording - W3C Working Draft, "RFC 6455". Disponível em: https://www.w3.org/TR/mediastreamrecording. [Acesso em 17 Dezembro 2017].

[22] W. Schools, "JSON," JavaScript Object Notation. Disponível em: https://www.w3schools.com/js/js_json_in tro.asp.

[23] A. Amarasinghe e P. Wimalaratne, "An Assistive Technology Framework for Communication with Hearing Impaired Persons", GSTF Journal on Computing (JoC), vol. 5, pp. 1-7, 2017.

[24] M. Mirzaei, S. Ghorshi e M. Mortazavi, "Helping deaf and hard-of-hearing people by combining augmented reality and speech technologies", 9th Intl Conf. Disability, Virtual Reality \& Associated Technologies, pp. 149-158, 2012.

[25] S. Cox, M. Lincoln, J. Tryggvason, M. Nakisa, M. Wells, M. Tutt e S. Abbott, "Tessa, a system to aid communication with deaf people", Proceedings of the fifth international ACM conference on Assistive technologies, pp. 205-212, 2002.

[26] R. S. S. Hernández, V. L. Ludeña, R. M. Maganto, S. B. L. Lutfi, J. F. López, R. C. Herralde e J. M. P. Muñoz, "Advanced Speech Communication System for Deaf People", 11th Annual Conference of the International Speech Communication Association, Interspeech 2010.

[27] M. S. F. Aranha, "Aranha, M. S. F. (2006). Saberes e práticas da inclusão: desenvolvendo competências para o atendimento às necessidades educacionais especiais de alunos cegos e de alunos com baixa visão", Coordenação geral

SEESP/MEC, 2006. 